\title{
Bayes Estimation of Topp-Leone Distribution Under Symmetric Entropy Loss Function Based on Lower Record Values
}

\author{
Lanping Li \\ Department of Basic Subjects, Hunan University of Finance and Economics, Changsha, China
}

Email address:

lilanping1981@163.com

To cite this article:

Lanping Li. Bayes Estimation of Topp-Leone Distribution Under Symmetric Entropy Loss Function Based on Lower Record Values. Science Journal of Applied Mathematics and Statistics. Vol. 4, No. 6, 2016, pp. 284-288. doi: 10.11648/j.sjams.20160406.16

Received: October 9, 2016; Accepted: October 20, 2016; Published: November 14, 2016

\begin{abstract}
This paper will study the estimation of parameter of Topp-Leone distribution based on lower record values. First, the minimum variance unbiased estimator and maximum likelihood estimator are obtained. Then the Bayes estimator is derived under symmetric loss function and further the empirical Bayes estimators is also obtained based on marginal probability density of record sample and maximum likelihood method. Finally, the admissibility and inadmissibility of a generally class of inverse linear estimators are also discussed.
\end{abstract}

Keywords: Admissibility, Bayes and Empirical Bayes Estimators, Record Values, Symmetric Entropy Loss Function

\section{Introduction}

Topp-Leone distribution was introduced by Topp and Leone in 1955 and used it to model some failure data. Ghitany and Kotz [1] pointed out Topp-Leone distribution is useful for modelling life-time phenomena. They studied some reliability measures of this distribution such as the hazard rate, mean residual life, reversed hazard rate, expected inactivity time, and their stochastic orderings. Al-Zahrani proposed a class of goodness-of-fit tests for the Topp-Leone distribution with estimated parameters and the proposed were based on the empirical distribution function, KolomogorovSmirnov, Cramer-von-Mises, etc. Sindhua [3] estimated the parameter of Topp-Leone distribution based on trimmed samples using Bayesian approach. Al-Zahrani and Alshomrani [4] studied the estimation of reliability of a stress-strength model in which the stress and strength samples are both distributed with Topp-Leone distribution. Bayoud [5] studied the estimation of the shape parameter of the Topp-Leone distribution from a view of classical and Bayesian approaches based on Type I censored samples. Feroze et al. [6] considered the Bayesian point and interval estimations of the two-component mixture of Topp-Leone distribution based on type I censored samples under a class of priors using four loss functions. El-Sayed et al. [7] used
Bayesian and non-Bayesian approach to estimate coefficient of variation of Topp-Leone distribution under an adaptive Type-II Progressive Censoring samples.

This paper is devoted to study the admissibility and inadmissibility of the estimators of the unknown parameter $\theta$ of the Topp-Leone distribution based on lower record values. The cumulative distribution function and probability density function of Topp-Leone distribution are given as follows, respectively ([8]):

$$
\begin{gathered}
F(x ; \theta)=\left(2 x-x^{2}\right)^{\theta}, 0<x<1 \\
f(x ; \theta)=2 \theta(1-x)\left(2 x-x^{2}\right)^{\theta-1}, 0<x<1
\end{gathered}
$$

Here $\theta>0$ is the unknown parameter.

Record values model, proposed by Chandler [9], is very related to the order statistics model, both of which are widely used in many real life applications involving data relating to weather, sports, economics, and life tests. sport, weather and life testing studies. Many authors have studied the statistical inference of various distributions based on record values and associated statistics. For example, Raqab [10] derived Exact expressions for single and product moments of three parameter generalized exponential distribution based record values. Soliman et al. [12] studied the Bayesian estimation in the context of record statistics values from the two-parameter 
Weibull distribution with respect to both symmetric loss function (squared error loss), and asymmetric loss function (LINEX) loss function. Jaheen [12] derived the Bayes estimators for the two unknown parameters of the Gompertz model using Laplace approximation under the squared error and LINEX loss functions. Ahmadi et al. [13] studied the Bayesian estimation and the admissibility of some estimators for the two parameters of the exponential distribution based on k-record values under LINEX loss function. More references can be found in [14-19].

The study of the admissible properties of estimators is an important topic in statistical inference. Yu and Sun [20] study the Bayes estimation and admissibility of the obtained estimators of Normal Distribution under q-Symmetric Entropy Loss Function. Bayoud [21] derived the Bayes and empirical Bayes estimates of the unknown parameter of Topp-Leone distribution under non informative and suitable conjugate priors. They proved that the proposed estimates are minimax and admissible. Zakerzadeh and Zahraie [22] studied the admissibility and inadmissibility of estimators of non-regular family under squared-log error loss function. Cao and kong [23] studied the admissibility of a class of linear estimators in a general multivariate linear model under balanced loss function.

The purpose of this paper is to study the estimation and admissible characters of estimators of the parameter of ToppLeone distribution. The organization of this paper is as follows. Section 2 derives the maximum likelihood (ML) estimator and minimum variance unbiased estimator of the parameter of Topp-Leone distribution. Section 3 discusses the Bayes and empirical Bayes estimators of the parameter . Section 4 studies the admissibility and inadmissibility of a class of inverse linear estimators. Finally, Section 5 gives the conclusion of this paper.

\section{ML and Minimum Variance Unbiased Estimation}

This section first recalls some definitions of lower record values, then derives the ML estimator and minimum variance unbiased estimator of the parameter of Topp-Leone distribution.

Definition 1. [24] Let $X_{1}, X_{2}, \cdots$ be a sequence of independent and identically distributed random variables. Suppose

$$
L(1)=1, L(n+1)=\min \left\{j: j>L(n), X_{j}<X_{L(n)}\right\}
$$

We say $X_{L(n)}, n=1,2, \cdots$ is a lower record value of this sequence.

Suppose $X_{L(1)}=x_{1}, X_{L(2)}=x_{2}, \cdots, X_{L(n)}=x_{n}$ be $\mathrm{n}$ lower record values from Topp-Leone distribution (1). $t=-\ln \left(2 x_{n}-x_{n}^{2}\right) \quad$ is the observation of $T=-\ln \left[2 X_{L(n)}-X_{L(n)}^{2}\right]$. When given the observation $\underline{x}=\left(x_{1}, x_{2}, \cdots, x_{n}\right) \quad$ of $\quad \underline{X}=\left(X_{L(1)}, X_{L(2)}, \cdots, X_{L(n)}\right)$. the likelihood function can be obtained as follows (Arnold et al. [23])

$$
\begin{aligned}
& l(\theta, \underline{x})=f\left(x_{n} ; \theta\right) \prod_{i=1}^{n-1} \frac{f\left(x_{i} ; \theta\right)}{F\left(x_{i} ; \theta\right)} \\
& =2 \theta\left(1-x_{n}\right)\left(2 x_{n}-x_{n}^{2}\right)^{\theta-1} \cdot \prod_{i=1}^{n-1} \frac{2 \theta\left(1-x_{i}\right)\left(2 x_{i}-x_{i}^{2}\right)^{\theta-1}}{\left(2 x_{i}-x_{i}^{2}\right)^{\theta}}
\end{aligned}
$$

Then we have

$$
l(\theta, \underline{x}) \propto \theta^{n} e^{-\left[-\ln \left(2 x_{n}-x_{n}^{2}\right)\right] \theta}=\theta^{n} e^{-\theta t}
$$

The MLE can be obtained by solving the log-likelihood equation

$$
\frac{d \ln l(\theta)}{d \theta}=0
$$

Then the MLE can be derived as

$$
\hat{\theta}_{M L E}=\frac{n}{T} .
$$

The probability density function of $X_{L(n)}$ is given by

$$
f_{n}\left(x_{n} ; \theta\right)=f\left(x_{n} ; \theta\right) \frac{R^{n-1}\left(x_{n} ; \theta\right)}{(n-1) !},
$$

Where $R(x ; \theta)=-\ln (F(x ; \theta))$.

Then we can get

$$
f_{n}\left(x_{n} ; \theta\right)=\frac{\theta^{n}}{(n-1) !} t^{n-1} e^{-\theta t}
$$

According to Eq. (5), the probability density function of $T$ can be easily derived as

$$
f_{T}(t)=\frac{\theta^{n}}{\Gamma(n)} t^{n-1} e^{-\theta t}
$$

It means that $T$ distributed with Gamma distribution $\Gamma(n, \theta)$.

Then

$$
E \frac{1}{T}=\frac{\theta}{n-1} .
$$

Further more, we have

$$
E\left(\hat{\theta}_{M L E}\right)=E\left(\frac{n}{T}\right)=\frac{n}{n-1} \theta,
$$

then

$$
E\left[\frac{n-1}{T}\right]=E\left[\frac{n-1}{n} \hat{\theta}_{M L E}\right]=\theta,
$$


thus $\frac{n-1}{T}$ is an unbiased estimator of $\theta$. According to Eq. (3), $T$ is a sufficient statistics for the first $\mathrm{n}$ lower record values. The estimator $\frac{n-1}{T}$ is the minimum variance unbiased estimator of $\theta$ by using Lehmann and Scheffe's theorem. That is

$$
\hat{\theta}_{U M V U}=\frac{n-1}{T} .
$$

\section{Bayes Estimation}

Prior distribution and loss function are very important parts in Bayesian analysis. Though squared error loss is the most often used loss function in Bayesian inference, but in recent years, more loss functions, such as LINEX loss, squared $\log$ error loss function, entropy loss function and symmetric entropy loss function are put forward and applied in Bayesian estimation. This section will derive the Bayes estimatior under the following symmetric entropy loss function (Zhao et al. [25])

$$
L(\hat{\theta}, \theta)=\frac{\hat{\theta}}{\theta}+\frac{\theta}{\hat{\theta}}-2
$$

The Bayes estimator under the symmetric entropy loss is denoted by $\hat{\theta}_{B}$, given by

$$
\hat{\theta}_{B}=\left[\frac{E(\theta \mid \underline{X})}{E\left(\theta^{-1} \mid \underline{X}\right)}\right]^{1 / 2} .
$$

Assume that the parameter $\theta$ has the Gamma prior distribution with prior parameters $\alpha>0$ and $\beta>0$, noted by, $\Gamma(\alpha, \beta)$. Then the prior probability density function of parameter $\theta$ is

$$
\pi(\theta \mid \alpha, \beta)=\frac{\beta^{\alpha}}{\Gamma(\alpha)} \theta^{\alpha-1} e^{-\beta \theta}, \theta>0
$$

Remark 1. The limiting case $\alpha, \beta \rightarrow 0$ of the prior Gamma distribution is non-informative prior $\pi(\theta) \propto \theta^{-1}$.

According to Eq. (3) and Eq. (10), using Bayesian theorem, we can get the posterior probability density function as follows:

$$
\begin{aligned}
h(\theta \mid x) & \propto l(\theta, \underline{x}) \cdot \pi(\theta ; \alpha, \beta) \\
& \propto \theta^{n} e^{-\theta t} \theta^{\alpha-1} e^{-\beta \theta} \\
& \propto \theta^{n+\alpha-1} e^{-(\beta+t) \theta}
\end{aligned}
$$

Then $h(\theta \mid x)$ is a posterior probability density of $\Gamma(n+\alpha, T+\beta)$, i.e. $\theta \mid \underline{X} \sim \Gamma(n+\alpha, T+\beta)$.

The Bayes estimator of $\theta$ under symmetric entropy loss function is

$$
\hat{\theta}_{B}=\left[\frac{E(\theta \mid \underline{X})}{E\left(\theta^{-1} \mid \underline{X}\right)}\right]^{1 / 2}=\left[\frac{(n+\alpha) /(T+\beta)}{(T+\beta) /(n+\alpha-1)}\right]^{1 / 2}
$$

Thus

$$
\hat{\theta}_{B}=\left[\frac{1}{\sqrt{(n+\alpha)(n+\alpha-1)}} T+\frac{\beta}{\sqrt{(n+\alpha)(n+\alpha-1)}}\right]^{-1}
$$

which belongs to the form $(c T+d)^{-1}$

Remark 2. When the parameter $\theta$ has the non-informative prior $\pi(\theta) \propto \theta^{-1}$, the posterior distribution of $\theta$ is $\Gamma(n, T)$. Then we obtain the generalized Bayes estimator

$$
\hat{\theta}_{*}=\frac{T}{\sqrt{n(n-1)}}
$$

Remark 3. When the prior parameter $\beta$ is unknown, we need estimate it using the empirical Bayes approach. From Eq. (3) and Eq. (10), the marginal probability density function of $\underline{X}=\left(X_{L(1)}, \cdots, X_{L(n)}\right)$ can be derived as

$$
\begin{aligned}
& m(\underline{x} \mid \beta)=\int_{0}^{\infty} f(\underline{x} ; \theta) \pi(\theta \mid \beta) d \theta \\
& =\int_{0}^{\infty} l(\theta, \underline{x}) \pi(\theta \mid \beta) d \theta \\
& \propto \int_{0}^{\infty} \theta^{n} e^{-\theta t} \frac{\beta^{\alpha}}{\Gamma(\alpha)} \theta^{\alpha-1} e^{-\beta \theta} d \theta \\
& \propto \frac{\beta^{\alpha}}{\Gamma(\alpha)} \frac{\Gamma(n+\alpha)}{(\beta+t)^{n+\alpha}}
\end{aligned}
$$

Based on $m(\underline{x} \mid \beta)$, The ML estimator of $\beta$ is $\hat{\beta}=\frac{\alpha}{n} T$.

Substitute $\hat{\beta}=\frac{\alpha}{n} T$ for $\beta$ in the Bayes estimator, then the empirical Bayes estimator is obtained as

$$
\hat{\theta}_{B}=\left[\frac{\sqrt{n+\alpha}}{n \sqrt{n+\alpha-1}} T\right]^{-1}
$$

Obviously, the empirical Bayes estimator $\hat{\theta}_{E B}$ also has the inverse linear form $(c T+d)^{-1}$.

\section{Admissibility of $(c T+d)^{-1}$}

The estimators obtained in Section 2 and Section 3 belong to the inverse linear estimators with the form $(c T+d)^{-1}$. This section will discuss the admissibility and inadmissibility of estimators. In the following discussion, we always supposes that:

(i) $X_{L(1)}=x_{1}, X_{L(2)}=x_{2}, \cdots, X_{L(n)}=x_{n}$ are $\mathrm{n}(\mathrm{n}>1)$ lower record values from Topp-Leone distribution (1).

(ii) Let $t$ be the observation of $T=-\ln \left[2 X_{L(n)}-X_{L(n)}^{2}\right]$, 
and $\underline{x}$ be the observation of $\underline{X}=\left(X_{L(1)}, X_{L(2)}, \cdots, X_{L(n)}\right)$.

(iii) $c^{*}=\frac{1}{\sqrt{n(n-1)}}$.

In the rest of this section, all obtained estimators will be compared on the basis of their risks under the entropy loss function. We will also study the conditions under which general inverse linear estimators are admissible in terms of risk function.

Theorem 1. If $0 \leq c<c^{*}$ and $d>0$, then the estimator $(c T+d)^{-1}$ is admissible.

Proof. From Eq. (12), it always true that the coefficient of $T$ is strictly between 0 and $c^{*}$ for any $\alpha>0$ and $\beta>0$, and the constant $\frac{\beta}{\sqrt{(n+\alpha)(n+\alpha-1)}}$ is strictly larger than 0 . Then the estimator $(c T+d)^{-1}$ is admissible for $0<c<c^{*}$ and $d>0$.

If $c=0, d>0$, then it is easy to verify that the estimator $(c T+d)^{-1}$ is also admissible since it is the unique Bayes estimator for which $R(\theta, d)=0$ when $\theta=d$.

Theorem 2. Let $(0,+\infty)$ be the parameter space and $[0,+\infty)$ be the action space. The inverse linear estimator $(c T+d)^{-1}$ is inadmissible under the symmetric entropy loss function (8) whenever one of the following conditions holds:

(i) $c<0$ or $d<0$;

(ii) $0<c \neq c^{*}, d=0$

(iii) $c>c^{*}$ and $d \geq 0$

Proof. (i) When $c<0$ or $d<0$, then we note that $(c T+d)^{-1}$ takes on negative values with positive probability. Then the estimator $(c T+d)^{-1}$ is dominated by the estimator $\max \left(0,(c T+d)^{-1}\right)$. Thus $(c T+d)^{-1}$ is inadmissible under case (i).

(ii) Under symmetric loss function, the risk function of the estimator $(c T)^{-1}$ is

$$
\begin{aligned}
R\left(\theta,(c T)^{-1}\right)= & E\left[\frac{(c T)^{-1}}{\theta}+\frac{\theta}{(c T)^{-1}}-2\right] \\
& =\frac{1}{c \theta} E\left[\frac{1}{T}\right]+c \theta E[T]-2 \\
& =\frac{1}{c \theta} \cdot \frac{\theta}{n-1}+c \theta \cdot \frac{n}{\theta}-2 \\
& =\frac{1}{c(n-1)}+c n-2
\end{aligned}
$$

Then derivative of the risk $R\left(\theta,(c T)^{-1}\right)$ with respect to $c$ is

$$
\frac{\partial}{\partial c} R\left(\theta,(c T)^{-1}\right)=-\frac{1}{c^{2}} \frac{1}{n-1}+n
$$

Then

$$
\frac{\partial}{\partial c} R\left(\theta,(c T)^{-1}\right)<0 \text { where } 0<c<c^{*}=\frac{1}{\sqrt{n(n-1)}},
$$

and

$$
\frac{\partial}{\partial c} R\left(\theta,(c T)^{-1}\right)>0, \text { where } c>c^{*}
$$

Thus the risk of the estimator $(c T)^{-1}$ is minimized at $c=c^{*}$. Then the estimator $(c T)^{-1}$ is dominated by the estimator $\left(c^{*} T\right)^{-1}$, and thus $(c T+d)^{-1}$ is inadmissible under case (ii).

(iii). Under the symmetric loss function, the difference of risk functions between the inverse linear estimator $(c T+d)^{-1}$ and $\left(c^{*} T+\frac{c^{*}}{c} d\right)^{-1}$ are as follows:

$$
\begin{aligned}
& R\left(\theta,(c T+d)^{-1}\right)-R\left(\theta^{*},\left(c^{*} T+\frac{c^{*}}{c} d\right)^{-1}\right) \\
& =E\left[\frac{(c T+d)^{-1}}{\theta}+\frac{\theta}{(c T+d)^{-1}}\right. \\
& \left.-\frac{\left(c^{*} T+\frac{c^{*}}{c} d\right)^{-1}}{\theta}-\frac{\theta}{\left(c^{*} T+\frac{c^{*}}{c} d\right)^{-1}}\right] \\
& =E\left[\frac{1}{\theta(c T+d)}+\theta(c T+d)\right. \\
& \left.-\frac{1}{\theta\left(c^{*} T+\frac{c^{*}}{c} d\right)}-\theta\left(c^{*} T+\frac{c^{*}}{c} d\right)\right] \\
& =\left(1-\frac{c^{*}}{c}\right)\left[\theta E(c T+d)-\frac{1}{\theta} E \frac{1}{c T+d}\right] \\
& \geq\left(1-\frac{c^{*}}{c}\right)\left(\theta E T-\frac{1}{\theta} E \frac{1}{T}\right) \\
& =\theta\left(1-\frac{c^{*}}{c}\right)\left(n-\frac{1}{n-1}\right)
\end{aligned}
$$

In the condition (iii), $1-\frac{c^{*}}{c}>0$, then

$$
R(\theta, c T+d)-R\left(\theta^{*}, c^{*} T+\frac{c^{*}}{c} d\right)>0
$$

Therefore, $R\left(\theta,(c T+d)^{-1}\right)$ is minimized at $c=c^{*}$. That is to say, $(c T+d)^{-1}$ is dominated by $\left(c^{*} T+d\right)^{-1}$ in this case. Then the estimator $(c T+d)^{-1}$ is inadmissible under the case (iii).

Remark 4. By Theorem 2, the ML estimator $\hat{\theta}_{M L E}$ and the empirical Bayes estimator $\hat{\theta}_{E B}$ are both inadmissible. They are both dominated by the Bayes estimator $\hat{\theta}_{*}=\left(c^{*} T\right)^{-1}$. 


\section{Conclusion}

This paper derived the ML estimator, minimum variance unbiased estimator, Bayes estimator and empirical Bayes estimator of the parameter of the Topp-Leone distribution on based on lower record values. These estimators all belong to inverse linear estimators with the form $(c T+d)^{-1}$. The admissibility and inadmissibility of $(c T+d)^{-1}$ are studied under different conditions. As a result, ML estimator and the minimum variance unbiased estimator are admissible.

\section{Acknowledgement}

This study is partially supported by Natural Science Foundation of Hunan Province (No. 2015JJ3030) and Foundation of Hunan Educational Committee (No.15C0228). The author also gratefully acknowledges the helpful comments and suggestions of the reviewers, which have improved the presentation.

\section{References}

[1] Ghitany M. E., Kotz S., Xie M., 2005. On some reliability measures and their stochastic orderings for the Topp-Leone distribution. Journal of Applied Statistics, 32(7):715-722.

[2] Al-Zahrani B., 2012. Goodness-of-fit for the Topp-Leone distribution with unknown parameters. Applied Mathematical Sciences, (125-128):6355-6363.

[3] Sindhua T. N., Saleemb M., Aslama M., 2013. Bayesian Estimation for Topp-Leone Distribution under Trimmed Samples. Journal of Basic and Applied Scientific Research 3(10):347-360.

[4] Al-Zahrani B., Alshomrani A., 2012. Inference on stressstrength reliability from Topp-Leone distributions. Journal of King Abdulaziz University-Science, 24(1):73-88.

[5] Bayoud H. A., 2015. Estimating the shape parameter of the Topp-Leone distribution based on Type I censored samples. Applicationes Mathematicae, 42(2):219-230.

[6] Feroze N., Aslam M., Saleem M., 2013. Statistical properties of two component mixture of Topp Leone distribution under a Bayesian approach. International Journal of Intelligent Technologies \& Applied Statistics, 6(3):403-404.

[7] El-Sayed M. A., Abd-Elmougod G. A., Abdel-Rahman E. O., 2015. Estimation for coefficient of variation of Topp-Leone distribution under adaptive Type-II progressive censoring scheme: Bayesian and non-Bayesian approaches. Journal of Computational \& Theoretical Nanoscience, 12(11):4028-4035.

[8] El-Sayed M. A., Abd-Elmougod G. A., Abdel-Khalek S., et al., 2013. Bayesian and non-Bayesian estimation of ToppLeone distribution based lower record values. 45(2):133-145.

[9] Chandler K. N., 1952. The distribution and frequency of record values, Journal of the Royal Statistical Society B, 14(2):220-228

[10] Raqab M. Z., 2002. Inferences for generalized exponential distribution based on record statistics. Journal of Statistical Planning \& Inference, 104(2):339-350.
[11] Soliman A. A, Ellah A. H. A., Sultan K. S., 2006. Comparison of estimates using record statistics from Weibull model: Bayesian and non-Bayesian approaches. Computational Statistics \& Data Analysis, 51(3):2065-2077.

[12] Jaheen Z. F., 2003. A Bayesian analysis of record statistics from the Gompertz model. Applied Mathematics \& Computation, 145(2):307-320.

[13] Ahmadi J., Doostparast M., Parsian A., 2005. Estimation and prediction in a two-parameter exponential distribution based on k-record values under LINEX loss function. Communication in Statistics-Theory and Methods, 34(4): 795805 .

[14] Amin E. A., 2012. Bayesian and non-Bayesian estimation from type I generalized logistic distribution based on lower record values, Journal of Applied Sciences Research, 2012(1):118-126.

[15] Selim M. A., 2012. Bayesian estimations from the twoparameter bathtub-shaped lifetime distribution based on record values, Pakistan Journal of Statistics \& Operation Research, 8(2):155-165.

[16] Zakerzadeh H., Jafari A. A., 2015, Inference on the parameters of two Weibull distributions based on record values, Statistical Methods \& Applications, 24(1):25-40.

[17] Arabi B. R., Arashi M., Tabatabaey S., 2014. Improved confidence intervals for the scale parameter of Burr XII model based on record values. Computational Statistics, 29(5): 11531173.

[18] Barranco-Chamorro I., Moreno-Rebollo J. L., JiménezGamero M. D., Alba-Fernández M. V., 2015. Estimation of the sample size based on record values. Mathematics \& Computers in Simulation, 55(118): 58-72.

[19] Algamal Z. Y., 2016. Using maximum likelihood ratio test to discriminate between the inverse Gaussian and Gamma distributions, International Journal of Statistical Distributions, 1 (1): 27-32.

[20] Du Y. J., Sun X. X., 2007. Estimation of scale parameter of normal distribution under q-Symmetric entropy loss function. Journal of Jilin University, 45(5):39-43.

[21] Bayoud H. A., 2015. Admissible minimax estimators for the shape parameter of Topp-Leone distribution. Communication in Statistics-Theory and Methods, 45(1):71-82.

[22] Zakerzadeh H., Zahraie S. H. M., 2015. Admissibility in nonregular family under squared-log error loss. Metrika, 78(2): 227-236.

[23] Cao, M. X., Kong, F. C., 2013. General admissibility for linear estimators in a general multivariate linear model under balanced loss function. Acta Mathematica Sinica, 29(29): 1823-1832.

[24] Arnold, B. C., Balakrishnan, N., Nagaraja, H. N., 1998. Records. New York: John Wiley \& Sons.

[25] Zhao S., Song Y., Song L., et al., 2007. Estimation of ordered means of two sample exponential distributions under symmetric entropy loss. Journal of Jilin University, 45(1):44-48. 